\title{
Testing the relationship between carbon-use efficiency and soil carbon formation in rhizosphere and detritusphere microbial communities
}

\author{
NOAH SOKOL ${ }^{1}$, MEGAN FOLEY ${ }^{2}$, BRUCE HUNGATE ${ }^{2}$, \\ MARY FIRESTONE ${ }^{3}$, STEVEN BLAZEWICZ ${ }^{1}$, ERIC \\ SLESSAREV $^{1}$, KATERINA ESTERA-MOLINA ${ }^{3}$, ALEX \\ GREENLON $^{3}$ AND JENNIFER PETT-RIDGE ${ }^{1}$ \\ ${ }^{1}$ Lawrence Livermore National Laboratory \\ ${ }^{2}$ Northern Arizona University \\ ${ }^{3}$ University of California Berkeley \\ Presenting Author: sokol1@1lnl.gov
}

Microbial residues are dominant ingredients of persistent soil organic matter (SOM). Via the 'microbial carbon pump,' plant carbon from litter and rhizodeposition is processed by the microbial community en route to forming SOM - particularly mineral-associated organic matter (MAOM). Yet, major uncertainty surrounds the microbial ecophysiological traits that regulate the microbial carbon pump, and how the relative importance of these traits varies in different soil hotspots (e.g. the rhizosphere and detritusphere) and under different moisture conditions. Most attention to date has focused on the role of microbial carbon-use efficiency (CUE), because this trait captures the partitioning of carbon to new microbial growth versus microbial respiration. For this reason, CUE has been widely posited to be positively related to MAOM formation. Yet there exists virtually no mechanistic evidence in support of this relationship to date.

To test this relationship, we conducted a 12 -week ${ }^{13} \mathrm{C}$ tracer study to track the movement of rhizodeposition and root detritus into microbial communities and SOM pools under normal moisture $(15 \pm 4.2 \%)$ or droughted $(8 \pm 2 \%)$ conditions. Using a continuous ${ }^{13} \mathrm{CO}_{2}$-labeling system, we grew the annual grass Avena barbata in controlled growth chambers and measured formation of MAOM from ${ }^{13} \mathrm{C}$-enriched rhizodeposition. In a second set of microcosms, we tracked the formation of MAOM derived from ${ }^{13} \mathrm{C}$-enriched $A$. barbata root detritus during 12 weeks of decomposition. We also measured a suite of microbial ecophysiological traits that we predict are important in soil carbon formation and persistence. Here, we present data on ${ }^{13} \mathrm{C}$ MAOM formation, as well as microbial CUE, measured via the ${ }^{18} \mathrm{O}-\mathrm{H}_{2} \mathrm{O}$ method.

Notably, we did not find support for a positive relationship between CUE and ${ }^{13} \mathrm{C}-\mathrm{MAOM}$ formation. Overall, we found the CUE-MAOM relationship was context-dependent, and that CUE was often a poor predictor of MAOM formation. We conclude that there may be other microbial ecophysiological traits (e.g. production of extracellular polymeric substances) that better predict MAOM formation than CUE alone. This implies that new research must focus on a broader suite of microbial traits including but not limited to CUE - to model the role of microbes in MAOM formation and SOM persistence. 